\title{
A Two-Step Approach toward Model-Free X-Ray Fluorescence Analysis of Layered Materials
}

\author{
Adrie D. Dane, ${ }^{\dagger}$ Hans A. van Sprang, ${ }^{\ddagger}$ and Lutgarde M. C. Buydens*,† \\ Katholieke Universiteit Nijmegen, Toernooiveld 1, 6525 ED Nijmegen, The Netherlands, and Philips Research, \\ Professor Holstlaan 4, 5656 AA Eindhoven, The Netherlands
}

\begin{abstract}
A two-step fundamental parameter method for model-free analysis of thin-layered materials by $\mathrm{X}$-ray fluorescence spectrometry is presented. In the first step, a genetic algorithm is used to obtain the number of layers and, for each layer, an estimate of the elementary concentrations and thickness. The second step is a gradient technique to refine this estimate. Good results are obtained for both relatively simple and more complex samples. The latter require extra depth information, which can be obtained from X-ray fluorescence measurements at various angles of detection.
\end{abstract}

In today's technology, an increasing use is made of materials consisting of thin layers with thicknesses that can vary from only several nanometers to a few micrometers. They are applied for their optical, mechanical, electrical, and/ or magnetic properties. An important application is the use of multiple thin layers of metal on silicon wafers in the integrated circuit technology. These materials can be quantitatively analyzed by X-ray fluorescence, where both layer thicknesses and elementary concentrations can be determined simultaneously.

Since adequate standards for layered materials scarcely exist, a so-called fundamental parameter method is most often used. Fundamental parameter methods are based on the calculation of theoretical X-ray intensities. First, the sample is described in terms of layers with estimated concentrations and thicknesses. This estimated model is iteratively adapted until measured and predicted intensities are consistent, according to an error criterion. The final concentrations and thicknesses obtained are assumed to represent the actual composition.

Currently applied fundamental parameter methods require an estimated sample model in which the number of layers and the distribution of the elements over the layers are fixed. The complexity of the sample and the optimization algorithm determine the required accuracy for the corresponding elementary concentrations and layer thicknesses.

This paper explores to what extent less adequate initial estimates can lead to good quantitative results. The method used is an adapted version of the method described in ref 1 . This new method consists of two optimization steps. In the first step, a genetic algorithm is used to obtain the number of layers, for each

\footnotetext{
† Katholieke Universiteit Nijmegen.

‡ Philips Research.

(1) Dane, A. D.; Timmermans, P. A. M .; van Sprang, H. A.; Buydens, L. M . C. Anal. Chem. 1996, 68, 2419.
}

layer the elements, and an estimate of the corresponding concentrations and layer thickness. The second step is a gradient technique to refine this extracted model. Good results are obtained for both relatively simple and more complex samples. The latter require X-ray fluorescence data measured at various angles of detection. This is discussed below in some detail.

\section{METHODS}

The complete description of a layered material consists of a qualitative model and corresponding quantitative parameters. The qualitative model is defined by the number of layers $M$ and, for each individual layer $m$, the number of elements $N_{m}$ and their identities. The quantitative parameters are the corresponding concentrations and layer thicknesses. In the remainder of this paper, we will use the term quantitative model for the combination of the qualitative model and the corresponding quantitative parameters. In all experiments described in this paper, the aim is to obtain this quantitative model from a set of measured (or simulated) X-ray intensities.

Because the method used in our preceding paper ${ }^{1}$ was not (yet) optimal, we could not conclude whether unsatisfactory results obtained in some of the experiments were caused by a lack of structural information in the intensities or by shortcomings in our method. In this paper, we use a more adequate method to answer this question. Since the method used is a modified version of the method already described in ref 1 , we merely discuss the most important modifications. These are the use of angular dependence of X-ray intensities, the genetic algorithm configuration, and the $M$ arquardt-Levenberg ${ }^{2}$ (gradient) technique for refining the quantitative parameters.

Angular Dependence of X-ray Intensities. In general, the intensities measured for thin-layer and multilayer samples contain structural information. This information is not necessarily sufficient to obtain the complete quantitative model, especially for complex multilayer samples. It is well known from the literature that the $X$-ray fluorescence intensities of a sample depend on the angles of incidence and detection with respect to the sample surface. This subject was reviewed in ref 3 , and it was concluded there that measurement of angular dependence of intensities can yield valuable additional information, especially for thin-layer and multilayer samples. In these samples, the angular dependence of

\footnotetext{
(2) Press, W, H.; Flannery, B. P.; Teukolski, S. A.; Vetterling, W. T. Numerical Recipes in C., The Art of Scientific Computing; Cambridge University Press: New York, 1988.

(3) de Boer, D. K. G. X-Ray Spectrom. 1989, 18, 119-129.
} 


\begin{tabular}{|c|c|c|c|c|c|c|c|}
\hline $0.2_{\left(\mathrm{Ti}_{1}\right)}$ & $0.4_{\left(\mathrm{As}_{1}\right)}$ & $0.4_{\left(\mathrm{Ag}_{1}\right)}$ & $\star\left(\mathrm{Si}_{1}\right)$ & $\star{ }^{\star}\left(\mathrm{Ti}_{2}\right)$ & $\star\left(\mathrm{As}_{2}\right)$ & $\star\left(\mathrm{Ag}_{2}\right)$ & $\star{ }^{\star}\left(\mathrm{Si}_{2}\right)$ \\
\hline$\star{ }^{\star}\left(\mathrm{Ti}_{3}\right)$ & $\star\left(\mathrm{As}_{3}\right)$ & $\star\left(\mathrm{Ag}_{3}\right)$ & $\star_{\left(\mathrm{Si}_{3}\right)}$ & $\star_{\left(\mathrm{Ti}_{4}\right)}$ & $\star\left(\mathrm{As}_{4}\right)$ & $\star\left(\mathrm{Ag}_{4}\right)$ & $1000_{\left(\mathrm{Si}_{4}\right)}$ \\
\hline $3_{\left(N_{1}\right)}$ & $\star\left(N_{2}\right)$ & $\star_{\left(N_{3}\right)}$ & $1_{\left(N_{4}\right)}$ & $2_{(M)}$ & & & \\
\hline
\end{tabular}

Figure 1. String representation of the simulated sim 1 sample $\left(M_{\text {repr }}\right.$ $=4)$. The actual string is binary encoded.

a given line is dependent not only on its wavelength but also on the locations of the emitting element.

In preliminary experiments, we investigated simulated samples of varying complexities. The results indicated that for relatively simple sample structures measurements under a single angle of detection yield enough information to obtain the quantitative model. For complex samples, the information in the intensities appeared insufficient. Therefore, for this type of data, we include the angular dependence by using the following two angles of detection: $\psi_{\text {out }}=10^{\circ}$ and $\psi_{\text {out }}=40^{\circ}$.

Genetic Algorithm Configuration. A good genetic algorithm configuration consists of a good string representation, a good fitness criterion, and a good choice of genetic operators with appropriate values for the corresponding control parameters. Compared with the genetic algorithm used in our preceding paper, ${ }^{1}$ we further optimized all three aspects. For general introductions in genetic algorithm methodology, the reader is referred to refs $4-7$.

(1) String Representation. In this paper, we represent an unknown sample, containing $N$ elements, by a number of layers

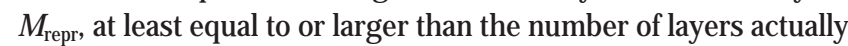
present $M_{\text {act }}$. The string representation consists of two parts: a qualitative part, which matches the qualitative sample model, and a quantitative part, which includes the corresponding quantitative parameters. Figure 1 depicts a string representation of sample sim 1: $\mathrm{Ti}_{0.2} \mathrm{As}_{0.4} \mathrm{Ag}_{0.4}(1 \mu \mathrm{m}) / \mathrm{Si}(1000 \mu \mathrm{m})$, in which $\mathrm{M}_{\text {repr }}=4$. The interpretation starts with the qualitative part (the bottom row): the number of layers $M$ and the number of elements $N_{m}$ for each present layer. In a normal sample, there are layers on top of a substrate, which is thicker than the escape depth of the radiation. Therefore, its thickness cannot be determined using XRF. In the experiments described below, this is also the case. For that reason, we use a fixed thickness. The interpretation is the same as for the other layers, except that the sum of the elemental thicknesses is not used as layer thickness. To avoid that the bottom layer is not present in generated trial solutions, the interpretation starts there. This is the reason that $\mathrm{N}_{4}$ (and not $\mathrm{N}_{2}$ ) is used in the qualitative part of Figure 1 . The $\mathrm{N}_{\mathrm{m}}$ values for the absent layers (those marked with $\star$ ) are not used. For each present layer, only the $\mathrm{N}_{\mathrm{m}}$ largest quantitative parameters (those not marked with $\star$ ) are used to obtain the quantitative model. The quantitative parameters are not plain concentrations but so-called elemental thicknesses, ${ }^{1}$ the products of concentration and corresponding layer thickness (in micrometers). The thickness of layer $m$ is the sum of the largest $\mathrm{N}_{\mathrm{m}}$ elemental thicknesses. Division of the

\footnotetext{
(4) Goldberg, D. E. Genetic Algorithms in Search, Optimization, and Machine Learning; Addison-Wesley: Reading, MA, 1989.

(5) M ichalewicz, Z. Genetic Algorithms + Data Structures = Evolution Programs Artificial Intelligence Series. Springer-Verlag: Berlin, 1992.

(6) Lucasius, C. B.; Kateman, G. Chem. Intell. Lab. Syst. 1993, 19, 1-33.

(7) Lucasius, C. B.; Kateman, G. Chem. Intell. Lab. Syst. 1994, 25, 99-146.
}

largest $\mathrm{N}$ elemental thicknesses by this thickness yields the corresponding $\mathrm{N}$ concentrations. Compared with more obvious string representations that consist of plain concentrations and layer thicknesses, this elemental thickness representation has an important advantage; i.e., the concentration sum in this representation is always equal to 1 . Therefore the genetic operators do not have to be restricted to yield concentration sums equal to 1 or that some form of scaling must be used for the string values to reach a concentration sum equal to 1 .

In ref 1 we used the same representation without the qualitative part. In that case, all $\mathrm{N} \times \mathrm{M}_{\text {repr }}$ elemental thicknesses had to converge to specific values; i.e., all elemental thicknesses of the elements present to their correct values and all elemental thicknesses of the nonpresent elements to zero. The latter was one of the problems in ref 1 ; in many solutions obtained, the elemental thicknesses of the nonpresent elements were close to zero but exact zeros, and thus exact solutions, were rarely obtained. Although we added extra parameters (the qualitative part) to the string, the number of parameters, which has to converge to a specific value, is usually less; in the Figure 1 example, 16 (all parameters in the first 2 rows) in the ref 1 representation and 7 in the representation used in this paper (the parameters not marked with $\star$ ). Another advantage of the Figure 1 representation is that this number is independent of the number of extra layers, whereas in the ref 1 representation, for each extra layer $\mathrm{N}$ (here 4), extra parameters must converge to specific values.

(2) Fitness Criterion. As error criterion, our genetic algorithm uses the elementwise relative error as described in ref 1.

$$
\text { error }=\frac{\sum_{n}^{N}\left(\sum_{j}^{J_{n}}\left|\frac{I_{\text {mea,nj }}-I_{\text {pred,nj }}}{I_{\text {mea,nj }}}\right| / J_{n}\right)}{N}
$$

The error is converted into a string fitness using fitness $=1$ / ( 1 + error). This results in a value between 0 (worst possible solution) and 1 (best possible solution). In two cases, we do not calculate the fitness in this manner. For both these exceptions, we directly set the fitness to 0 , the worst possible solution. In the first exception, two adjacent layers have the same quantitative elemental composition. This avoids degenerate solutions since these solutions are the same as those in which the two layers are taken together. The second exception is the case in which a predicted line is absent, $I_{\text {pred,nj }}=0$, while the corresponding measured (or simulated) line is present, $I_{\text {mea,nj }}>0$. This avoids solutions in which an element is absent.

(3) Genetic O perator Configuration. The genetic operators and their corresponding control parameters are the same for each sample and as such they are part of the method. Compared with ref 1 , we made several changes. The most important adaptation is the use of crowding, ${ }^{8,9}$ sharing, ${ }^{10}$ and bit-complement selection ${ }^{11,12}$ operators. We found that use of these operators, which are designed to prevent preliminary convergence, is essential for

(8) de Jong, K. A. Artificial genetic adaptive systems. Technical Report 76-7; Department of Computer Science, University of Pittsburgh, Pittsburgh, 1976.

(9) de Jong, K. A.; Sarma, J. Generation gaps revisited. In F oundations of Genetic Algorithms 2; Whitley, L. D., Ed.; M organ Kaufmann Publishers: San M ateo, CA, 1993.

Analytical Chemistry, Vol. 71, No. 20, October 15, 1999 


\section{Table 1. XRF Data}

$\begin{array}{cc}\text { sample } & \text { composition } \\ \text { sim } 1 & \mathrm{Ti}_{0.2} \mathrm{As}_{0.4} \mathrm{Ag}_{0.4}(1 \mu \mathrm{m}) / \mathrm{Si}(1000 \mu \mathrm{m}) \\ \operatorname{sim} 2 & \mathrm{~Pb}_{0.2} \mathrm{Zr}_{0.4 \mathrm{Ti}} \mathrm{Ti}_{0.4}(1 \mu \mathrm{m}) / \mathrm{Pt}(\mathrm{Im}) / \\ \operatorname{sim} 3 & \mathrm{~Pb}_{0.2 \mathrm{Z} \mathrm{Zr}_{0.4 \mathrm{Ti}} / \mathrm{Si}(1000 \mu \mathrm{m})}(0.1 \mu \mathrm{m}) / \mathrm{Pt}(0.1 \\ & \left.\mu \mathrm{m}) / \mathrm{Ti}_{0.1} \mu \mathrm{m}\right) / \mathrm{Si}(1000 \mu \mathrm{m}) \\ \text { mea } 1 & \mathrm{Fe}_{0.4811} \mathrm{~Tb}_{0.4656} \mathrm{Co}_{0.0534} \\ & (0.047 \mu \mathrm{m}) / \mathrm{Si}_{1.0}(>1000 \mu \mathrm{m})\end{array}$

(0. $047 \mu \mathrm{m}) / \mathrm{Si}_{1.0}(>1000 \mu \mathrm{m})$

\author{
lines \\ $\psi_{\text {out }}$ \\ $\mathrm{AgK} \alpha, \mathrm{AgL} \alpha, \mathrm{AsK} \alpha, \mathrm{AsL} \alpha$, \\ $\operatorname{SiK} \alpha, \operatorname{TiL} \alpha, \operatorname{TiK} \alpha$ \\ $\operatorname{SiK} \alpha, \operatorname{TiK} \alpha, \operatorname{TiL} \alpha, \operatorname{PbL} \alpha, \operatorname{PbM} \alpha$, \\ $\operatorname{PtL} \alpha, \operatorname{PtM} \alpha, Z r K \alpha, Z r L \alpha$ \\ SiK $\alpha$, TiK $\alpha$, TiL $\alpha$, PbL $\alpha, \operatorname{PbM} \alpha$, \\ $\operatorname{PtL} \alpha, \operatorname{PtM} \alpha, \operatorname{ZrK} \alpha, \operatorname{ZrL} \alpha$ \\ $\operatorname{SiK} \alpha, \operatorname{FeK} \alpha, \operatorname{CoK} \beta, \operatorname{TbL} \alpha$
}

the performance of the method. The configuration used by our method can be obtained as Supporting Information.

Marquardt-Levenberg Optimization. In a genetic algorithm application, the user is free to choose an error criterion. This in contrast to the $M$ arquardt-Levenberg algorithm, ${ }^{2}$ which by definition minimizes $\chi^{2}$. Here, best results are obtained with

$$
\chi^{2}=\sum_{n=1}^{N} \sum_{j=1}^{J_{n}}\left(\frac{I_{\text {mea,nj }}-I_{\text {pred,nj }}}{I_{\text {mea,nj }}}\right)^{2}
$$

$\chi^{2}$ in this manner is a quadratic relative error, which gives equal weight to all intensities.

\section{MATERIALS}

XRF Data. The experiments described in this paper concern one measured and three simulated data sets. The samples and corresponding lines are shown in Table 1. The actual intensities are available as Supporting Information. $\psi_{\text {out }}$ denotes the emerging angles used. The other conditions are the same for all data: chromium tube, $26^{\circ}$ anode angle, $0.5-\mathrm{mm}$ beryllium window, 60 $\mathrm{kV}, 50 \mathrm{~mA}$, no filter, incident angle $57^{\circ}$.

The simulated data sets ( $\operatorname{sim} 1-\operatorname{sim} 3)$ were created by calculating theoretical intensities for the corresponding sample composition. For this we used the fundamental parameter method for multilayer materials by De Boer ${ }^{13}$ and a primary spectral distribution from an algorithm by Pella et al.14,15 Since our computer program uses the same noise-free calculations, for these data exact solutions are possible: $\chi^{2}=0$. The layer densities $\rho_{\mathrm{m}}$ required were calculated from the elemental concentrations $C_{n m}$ and the elemental densities $\rho_{n}$ using, $\rho_{m}=1 /\left(\sum_{n=1}^{N_{m}}\left(C_{n m} / \rho_{n}\right)\right)$. The densities obtained in this manner are only an approximation of the correct values. However, they determine the values of the thicknesses obtained in the experiments. Therefore, normally it is better to use the mass thickness (product of layer density and thickness) instead of the normal thickness for the presentation

(10) Goldberg, D. E.; Richardson, J. Genetic algorithms with sharing for multimodal function optimization, in genetic algorithms and their applications. In Genetic algorithms and their applications: Proceedings of the second international conference on genetic algorithms; Grefenstette, J. J., Ed.; L. Erlbaum Associates: Hillsdale, NJ, 1987; p 41.

(11) Grefenstette, J. J. Deception considered harmful. In F oundations of Genetic Algorithms 2; Whitley, L. D., Ed.; M organ Kaufmann Publishers: San M ateo, CA, 1993.

(12) Louis, S. J.; Rawlins, G. J. E. Syntactic analysis of convergence in genetic algorithms. In Foundations of Genetic Algorithms 2; Whitley, L. D., Ed.; M organ Kaufmann Publishers: San M ateo, CA, 1993.

(13) de Boer, D. K. G. X-Ray Spectrom. 1990, 19, 145-154.

(14) Pella, P. A.; Feng, L.; Small, J. A. X-Ray Spectrom. 1985, 14, 125-135.

(15) Pella, P. A.; Feng, L,; Small, J. A. X-Ray Spectrom. 1991, 20, 109-110. of results. For the clarity of this paper and because the calculated densities are correct for the simulated data used, we use the normal thickness.

The only measured data used in this study are the mea 1 data. The mea 1 intensities are recorded using a Philips PW1404 spectrometer. This spectrometer is not equipped to measure at more than one angle of detection. Therefore, no measured data are used to investigate that aspect. In order to compare measured intensities with theoretical intensities a so-called instrument factor must be applied. The procedure is described in ref 16 . The concentrations and layer thickness for the top layer are obtained by analysis with FP-M ULTI, ${ }^{16}$ which is a commercial fundamental parameter program. The thickness of the second layer is not given since it is larger than the escape depth of the radiation. B oth the fundamental parameter calculations for multilayer materials and the primary spectral distribution in FP-MULTI are the same as those used in our program. The optimization method in FP-M ULTI is a Gauss-Newton optimization, which is a gradient technique. In FP-MULTI, the user must supply the layer densities. These densities are kept fixed during the optimization. Here the following values are used: $\rho_{\mathrm{m}}=8.0 \mathrm{~g} / \mathrm{cm}^{3}$ for the top layer and $\rho_{\mathrm{m}}=2.33$ $\mathrm{g} / \mathrm{cm}^{3}$ for the bottom layer.

Computer Program. The computer program was written in ANSI-C and compiled using the GNU C compiler version 2.7.2. For the genetic algorithm part, we used the GA3 library. ${ }^{17}$ The program was run under Linux on a PC with a 133-M Hz Pentium processor. Using this hardware, the running times were roughly $2 \mathrm{~h}$ for the four-layer samples and $20 \mathrm{~min}$ for the two-layer samples. These running times can be reduced, since in most cases the genetic algorithm converged to its final solution in far less than the total number of evaluations we used as stop criterion.

\section{COMPUTER EXPERIMENTS}

The computer experiments described in this paper are summarized in Table 2. The columns are, from left to right, the sample, the number of intensities J, the number of layers actually present $M_{\text {act, }}$, the number layers used in the representation $M_{\text {repr, }}$, the number of quantitative parameters when the qualitative model is correct $\mathrm{Q}_{\text {act, }}$ and the number of quantitative parameters used in the representation $Q_{\text {repr. }}$ All experiments are carried out in replicates of 10 by initiating the genetic algorithm runs with 10 different random seeds. The genetic algorithm configuration, which is the same for all experiments, can be obtained as

(16) de Boer, D. K. G.; Borstrok, J. J. M .; Leenaerts, A. J. G.; van Sprang, H. A. X-Ray Spectrom. 1993, 22, 1-6.

(17) Dane, A. D. Chemometrical contributions to analytical X-ray spectrometry. Ph.D. Thesis, Katholieke Universiteit Nijmegen, January 1999. 


\section{Table 2. Computer Experiments}

\begin{tabular}{lccccc} 
sample & $J$ & $M_{\text {act }}$ & $M_{\text {repr }}$ & $Q_{\text {act }}$ & $Q_{\text {repr }}$ \\
sim 1 & 7 & 2 & 4 & 3 & 16 \\
mea 1 & 4 & 2 & 4 & 3 & 16 \\
sim 2 & 18 & 4 & 6 & 5 & 30 \\
sim 3 & 18 & 4 & 6 & 5 & 30 \\
\hline
\end{tabular}

\begin{tabular}{lll}
\hline \multicolumn{2}{c}{ Table 3. Results Sample sim $\mathbf{1}$} & \\
parameter & comp & \multicolumn{1}{c}{$\mathrm{A}$} \\
$\mathrm{Ti}_{1}(\%)$ & 20.0 & 20.0 \\
$\mathrm{As}_{1}(\%)$ & 40.0 & 40.0 \\
$\mathrm{Ag}_{1}(\%)$ & 40.0 & 40.0 \\
$\mathrm{Si}_{1}(\%)$ & & 0.0 \\
$\mathrm{~d}_{1}(\mu \mathrm{m})$ & 1.0 & 1.0 \\
$\operatorname{layer}_{2}$ & & - \\
$\operatorname{layer}_{3}$ & & - \\
$\mathrm{Ti}_{4}(\%)$ & & - \\
$\mathrm{As}_{4}(\%)$ & & - \\
$\mathrm{Ag}_{4}(\%)$ & & 100.0 \\
$\mathrm{Si}_{4}(\%)$ & 100.0 & 1000.0 \\
$\mathrm{~d}_{4}(\mu \mathrm{m})$ & 1000.0 & 10 \\
$\mathrm{no}$. of exps & & $0.028-0.110$ \\
$\mathrm{error}$ & & $1.01 \times 10^{-12}$ \\
$\chi_{2}$ & & \\
\hline
\end{tabular}

Supporting Information. A genetic algorithm optimizes each parameter between a lower and an upper bound. For each qualitative parameter, the lower bound is 1 . The upper bounds on the number of elements in each layer and the number of layers are $\mathrm{N}$ and $\mathrm{M}_{\text {repr, }}$ respectively. The lower bounds on the elemental thicknesses are 0 . The upper bounds are twice the corresponding layer thickness for all present layers and the same as those used for the top layer for the nonpresent layers.

$Q_{\text {act }}$ is the number of (quantitative) parameters that would be used in a traditional fundamental parameter method, where the qualitative sample model is correct. This is the summation of the numbers of elements for each layer minus 1 (the bottom layer has a fixed thickness). The total number of parameters in our experiments is the number of quantitative parameters $Q_{\text {repr }}$ plus the number of layers (with nonfixed thickness). Normally, it is believed impossible to find unambiguous solutions when there are more (quantitative) parameters than XRF lines. This is the case in all experiments. We will discuss this subject further using the experimental results (see the Results section).

In the experiments on the mea 1 sample, the results are compared with FP-M ULTI results. To make this comparison fair, the same fixed densities are used: $\rho_{\mathrm{m}}=2.33 \mathrm{~g} / \mathrm{cm}^{3}$ for the bottom layer and $\rho_{\mathrm{m}}=8.0 \mathrm{~g} / \mathrm{cm}^{3}$ for all other layers. This in contrast to all other experiments, which calculate the layer densities as described in the XRF data section.

In all experiments, the calculated $X$-ray intensities consist only of primary fluorescence in the genetic algorithm part and of both primary and secondary fluorescence in the gradient part.

\section{RESULTS}

Tables 3-6 provide the experimental results. Each quantitative model obtained is denoted by a capital letter. The structural information is presented in terms of concentrations and thicknesses. These concentrations and thicknesses are recalculated from the corresponding elemental thicknesses used in optimization. A minus sign ( - ) denotes that in the qualitative model found by the genetic algorithm the corresponding element or layer is absent. The actual composition for the simulated samples is denoted by "comp".

There are three statistics for each of the quantitative models obtained. "N o. of exps" is the number of replicates that converged to that particular quantitative model. "Error" is the best ever elementwise relative error obtained in the first optimization step (the genetic algorithm). Although the end result obtained in the replicate experiments is the same for each column, the intermediate results may differ, which is expressed by an error range. $\chi^{2}$ is the eq 2 calculated value obtained after $M$ arquardt-Levenberg optimization. There are small differences in the less significant decimals of replicates that converged to the same quantitative model. The tabulated value is the largest corresponding $\chi^{2}$ value obtained.

The results for sample sim 1 are given in Table 3. All 10 experiments converged to $A$, which represents the exact solution. Although in the qualitative model silicon is present in the top layer, the corresponding concentration has converged to zero in the second optimization step. The experiment indicates that X-ray measurements at $\psi_{\text {out }}=40^{\circ}$ can contain enough information to obtain the quantitative structure for relatively simple samples.

Table 4 shows the results for sample mea 1 . Nine out of the 10 replicate experiments converged to $A$ or $B$ ( $A$ and $B$ represent the same quantitative model), which both represent the same quantitative solution as obtained with FP-M ULTI. In B, silicon is present in the top layer; again, the corresponding concentration has converged to zero in the second optimization step. $\mathrm{C}$ is a degenerate solution and not a local optimum since the corresponding error is in the same range as the errors for $A$ and $B$. Upon further inspection we noticed a continuum of degenerate three-layer solutions. The information required to make an unambiguous decision about the actual structure is not present in the intensities. A way to avoid these degenerate solutions is to measure extra lines or to use two angles of detection.

Table 5 depicts the results for the sim 2 data. We also analyzed this sample with only the intensities simulated at $\psi_{\text {out }}=40^{\circ}$. In that case, all 10 replicates converged to wrong solutions. Here intensities are used at both $\psi_{\text {out }}=40^{\circ}$ and $\psi_{\text {out }}=10^{\circ}$, and the exact solution (represented by $A, B$, and $C$ ) is obtained in 8 out of the 10 replicates. In $B$, there is platinum in the third layer, and in $\mathrm{C}$, there is platinum in the top layer. However, as can be seen in Table 5 , both concentrations have converged to 0 during the optimization. The two wrong models ( $D$ and $E$ ) are local optimums because the errors are much larger than those for $A, B$, and $C$. The most important differences between $D$ and the correct structure are the second (platinum) layer, which is $1.2 \mu \mathrm{m}$ instead of $1.0 \mu \mathrm{m}$, and the absence of the third (titanium) layer. The difficulty in obtaining the third layer is that titanium is also present in the first layer. The third layer almost does not contribute to the four titanium intensities. Only for the Ti K $\alpha$ intensity at $\psi_{\text {out }}$ $=40^{\circ}$ is there a minor contribution. For $\mathrm{Ti} \mathrm{K} \alpha$ at $\psi_{\text {out }}=10^{\circ}$ and both TiL $\alpha$ intensities, there is practically no contribution of the third layer, because all radiation is absorbed by the sample. The reason that the second layer is $1.2 \mu \mathrm{m}$ instead of $1.0 \mu \mathrm{m}$ is that both the thicknesses of the second and third layers largely 


\section{Table 4. Results Sample mea 1}

\begin{tabular}{|c|c|c|}
\hline parameter & FP-M ULTI & A \\
\hline $\mathrm{Fe}_{1}(\%)$ & 48.1 & 48.1 \\
\hline $\mathrm{Tb}_{1}(\%)$ & 46.6 & 46.6 \\
\hline $\mathrm{CO}_{1}(\%)$ & 5.3 & 5.3 \\
\hline $\mathrm{Si}_{1}(\%)$ & & - \\
\hline $\mathrm{d}_{1}(\mu \mathrm{m})$ & 0.047 & 0.047 \\
\hline $\mathrm{Fe}_{2}(\%)$ & & - \\
\hline $\mathrm{Tb}_{2}(\%)$ & & - \\
\hline $\mathrm{CO}_{2}(\%)$ & & - \\
\hline $\mathrm{Si}_{2}(\%)$ & & - \\
\hline$d_{2}(\mu \mathrm{m})$ & & - \\
\hline layer $_{3}$ & & - \\
\hline $\mathrm{Fe}_{4}(\%)$ & & - \\
\hline $\mathrm{Tb}_{4}(\%)$ & & - \\
\hline $\mathrm{CO}_{4}(\%)$ & & - \\
\hline $\mathrm{Si}_{4}(\%)$ & 100.00 & 100.00 \\
\hline $\mathrm{d}_{4}(\mu \mathrm{m})$ & 1000.00 & 1000.00 \\
\hline no. of exps & & 7 \\
\hline error & & $\begin{array}{l}0.0043-0.0051 \\
233 \times 10^{-4}\end{array}$ \\
\hline
\end{tabular}

\begin{tabular}{ll} 
& \multicolumn{1}{c}{$C$} \\
& 50.5 \\
& 49.5 \\
& - \\
& - \\
& 0.044 \\
& 11.0 \\
& - \\
& 88.9 \\
& 0.003 \\
& - \\
& - \\
& - \\
& - \\
& - \\
00 & 100.00 \\
& 1000.00 \\
0.00 & 1 \\
& 0.0038 \\
$41-0.0336$ & $2.31 \times 10^{-4}$ \\
\hline $10^{-4}$ &
\end{tabular}

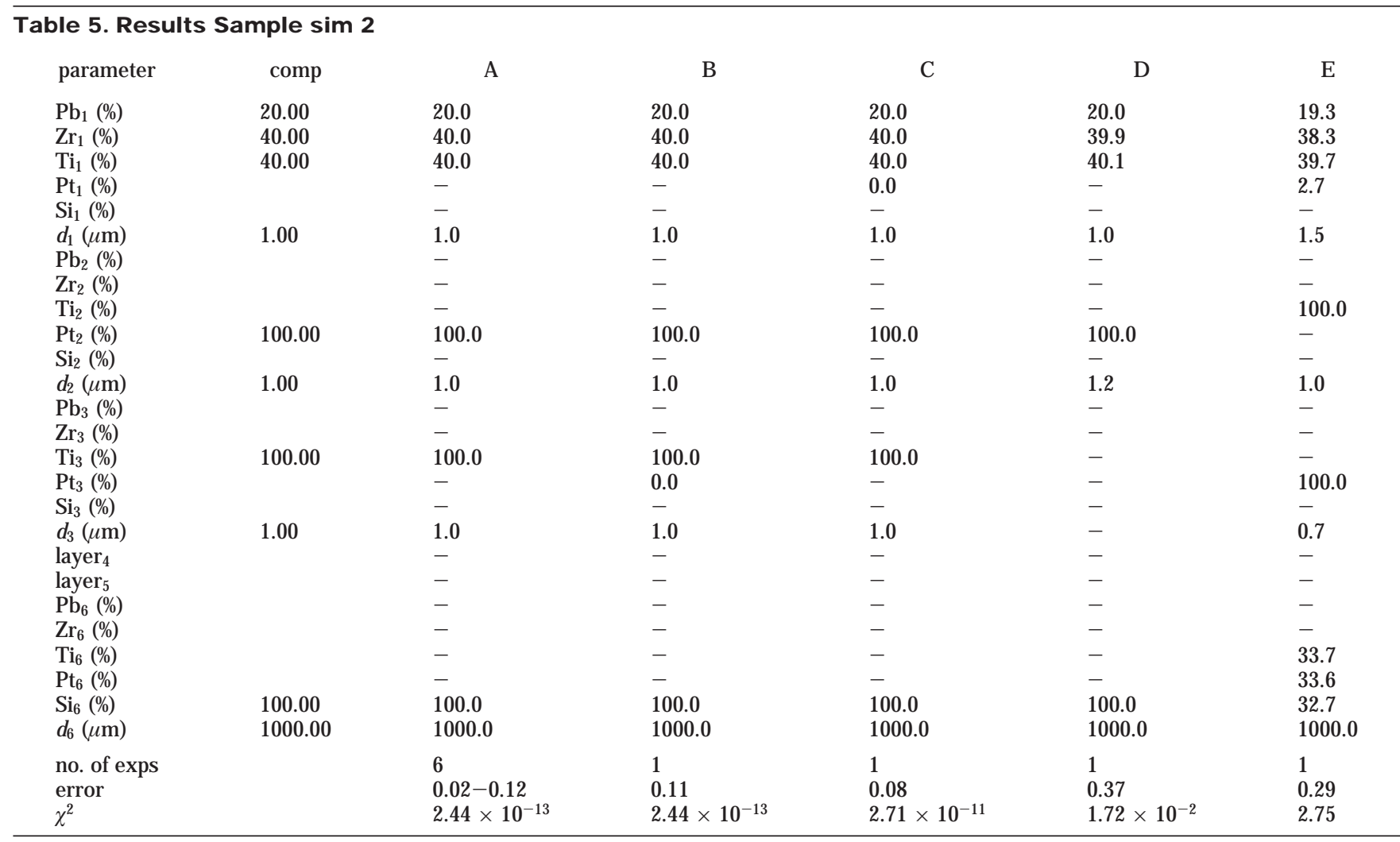

influence the Si $K \alpha$ intensities. When the third layer is absent, and the platinum layer is $1.0 \mu \mathrm{m}$, the Si $\mathrm{K} \alpha$ intensities are more than 3 times larger than the simulated intensities for sim 2 . When the platinum layer is $1.2 \mu \mathrm{m}$, the Si K $\alpha$ intensities are fitted much better. Although the platinum intensities are fitted somewhat worse, the corresponding $\chi^{2}$ value is much smaller. $\mathrm{E}$ is very different from the correct structure, which explains the large $\chi^{2}$ value. The intermediate error is somewhat smaller than that for $D$. For this reason, $D$ and $E$ are comparable solutions after genetic algorithm optimization. $E$ is a local optimum from which the second optimization step cannot escape, since the qualitative model is fixed. Because the structure is so different from the correct solution, it is not so easy but also not relevant to discuss specific differences.

Table 6 shows the results obtained for the sim 3 sample. Only in two experiments, is the exact composition ( $A$ and $B$ ) found. Extra parameters are present in both qualitative models. However, as can be seen in Table 6 , all these parameters have converged to 0 in the optimization. Much of the information about the qualitative model is explained by the attenuation of fluorescent radiation by the layers on top of the layer where the radiation originates. When the layers are thinner, the attenuation effects are less visible in the intensities, which explains why $\operatorname{sim} 3$ is more difficult to analyze than $\operatorname{sim} 2$. 


\section{Table 6. Results Sample sim 3}

\begin{tabular}{|c|c|c|c|c|c|c|c|c|}
\hline parameter & comp & A & B & C & D & $E$ & $\mathrm{~F}$ & G \\
\hline $\mathrm{Pb}_{1}(\%)$ & 20.0 & 20.0 & 20.0 & 15.8 & 11.6 & 11.7 & 20.8 & - \\
\hline $\mathrm{Zr}_{1}(\%$ & 40.0 & 40.0 & 40.0 & 39.4 & 22.5 & 22.8 & 41.4 & - \\
\hline $\mathrm{Ti}_{1}(\%)$ & 40.0 & 40.0 & 40.0 & 41.2 & 35.7 & 35.9 & 37.9 & - \\
\hline $\mathrm{Pt}_{1}(\%)$ & & 0.0 & 0.0 & 3.6 & 30.2 & 29.5 & - & 100.00 \\
\hline $\mathrm{Si}_{1}(\%)$ & & 0.0 & 0.0 & - & - & 0.0 & - & - \\
\hline $\mathrm{d}_{1}(\mu \mathrm{m})$ & 0.10 & 0.10 & 0.10 & 0.09 & 0.16 & 0.16 & 0.10 & 0.00 \\
\hline $\mathrm{Pb}_{2}(\%)$ & & - & - & 40.9 & - & - & - & 20.7 \\
\hline $\mathrm{Zr}_{2}(\%$ & & - & - & 31.4 & - & - & - & 41.6 \\
\hline $\mathrm{Ti}_{2}(\%)$ & & - & - & - & - & - & 12.8 & 37.7 \\
\hline $\mathrm{Pt}_{2}(\%)$ & 100.0 & 100.0 & 100.0 & 0.9 & 100.0 & 100.0 & 87.2 & - \\
\hline $\mathrm{Si}_{2}(\%)$ & & - & - & 26.8 & - & - & - & 0.0 \\
\hline$d_{2}(\mu \mathrm{m})$ & 0.10 & 0.10 & 0.10 & 0.02 & 0.09 & 0.09 & 0.18 & 0.09 \\
\hline $\mathrm{Pb}_{3}(\%)$ & & - & - & - & - & - & - & 0.1 \\
\hline $\mathrm{Zr}_{3}(\%)$ & & 0.0 & - & - & - & - & - & 0.0 \\
\hline $\mathrm{Ti}_{3}(\%)$ & 100.0 & 100.0 & 100.0 & - & - & - & - & 12.9 \\
\hline $\mathrm{Pt}_{3}(\%)$ & & - & 0.0 & 100.0 & - & - & - & 87.0 \\
\hline $\mathrm{Si}_{3}(\%)$ & & - & 0.0 & 0.1 & - & - & 100.0 & 0.1 \\
\hline $\mathrm{d}_{3}(\mu \mathrm{m})$ & 0.10 & 0.10 & 0.10 & - & - & - & 1000.0 & 0.18 \\
\hline $\mathrm{Pb}_{4}(\%)$ & & - & - & - & - & - & - & - \\
\hline $\mathrm{Zr}_{4}(\%)$ & & - & - & - & - & - & - & - \\
\hline $\mathrm{Ti}_{4}(\%)$ & & - & - & 100.0 & - & - & - & - \\
\hline $\mathrm{Pt}_{4}(\%)$ & & - & - & - & - & - & 100.0 & - \\
\hline $\mathrm{Si}_{4}(\%$ & & - & - & - & - & - & - & - \\
\hline $\mathrm{d}_{4}(\mu \mathrm{m})$ & & - & - & 0.1 & - & - & 57.5 & - \\
\hline layer $_{5}$ & & - & - & - & - & - & - & - \\
\hline $\mathrm{Pb}_{6}(\%)$ & & - & - & - & - & - & - & - \\
\hline $\mathrm{Zr}_{6}(\%)$ & & - & - & - & - & - & - & - \\
\hline $\mathrm{Ti}_{6}(\%)$ & & - & - & - & - & - & - & - \\
\hline $\mathrm{Pt}_{6}(\%)$ & & - & - & - & - & - & - & - \\
\hline $\begin{array}{l}\mathrm{Si}_{6}(\%) \\
\mathrm{d}_{6}(\mu \mathrm{m})\end{array}$ & $\begin{array}{l}100.0 \\
1000.0\end{array}$ & $\begin{array}{l}100.0 \\
1000.0\end{array}$ & $\begin{array}{l}100.0 \\
1000.0\end{array}$ & $\begin{array}{l}100.0 \\
1000.0\end{array}$ & $\begin{array}{l}100.0 \\
1000.0\end{array}$ & $\begin{array}{l}100.0 \\
1000.0\end{array}$ & $\begin{array}{l}100.0 \\
1000.0\end{array}$ & $\begin{array}{l}100.0 \\
1000.0\end{array}$ \\
\hline no. of exps & & 1 & 1 & 1 & 4 & 1 & 1 & 1 \\
\hline error & & 0.063 & 0.048 & 0.057 & $0.064-0.074$ & 0.093 & 0.071 & 0.222 \\
\hline$\chi^{2}$ & & $2.08 \times 10^{-12}$ & $4.33 \times 10^{-11}$ & $7.25 \times 10^{-3}$ & $1.79 \times 10^{-1}$ & $1.79 \times 10^{-1}$ & $2.77 \times 10^{-2}$ & $2.75 \times 10^{-2}$ \\
\hline
\end{tabular}

The results, especially those for $\operatorname{sim} 1$ and $\operatorname{sim} 2$, show that good reproducible results can be obtained, even though the number of XRF lines J is smaller than the number of (quantitative) parameters. This indicates that the lines contain sufficient information. The number of quantitative parameters, which is optimized in the second step, is smaller than $\mathrm{J}$ in all experiments (or equal to $\mathrm{J}$, in C for mea 1 see Table 4). We observed that already in the early stages of the genetic algorithm runs the best solutions at that stage contained a number of quantitative parameters, which was equal or only somewhat larger than the number of quantitative parameters in the end solutions. What can be found in literature $\mathrm{e}^{4,6,7}$ is that genetic algorithms can very effectively locate the most promising regions of a search space. This is supported by our results, although we could not find an exact theoretical explanation.

The simulated data used in this paper are of increasing complexity. Correct solutions are obtained in $100 \%$ (10 out of 10) of the experiments for $\operatorname{sim} 1,80 \%$ ( 8 out of 10 ) for $\operatorname{sim} 2$, and $20 \%$ ( 2 out of 10 ) for $\operatorname{sim} 3$. For the two experiments on sim 2, which yield wrong solutions (Table $5, D$ and $E$ ), the intermediate errors obtained in the genetic algorithm step are clearly larger than those for the correct solutions. For $\operatorname{sim} 3$, this is the case in only one (Table 6, G) of the eight experiments that yield wrong solutions. These three wrong solutions are caused by preliminary convergence of the genetic algorithm. Since preliminary convergence takes place only in these three experiments, the genetic algorithm configuration seems robust.
In seven of the eight experiments on sim 3, which converge to wrong solutions (Table $6, \mathrm{C}-\mathrm{F}$ ), the intermediate errors are comparable to those of the correct solutions ( $A$ and $B$ ). The errors are explained by the limited search precision, which is a property of genetic algorithms. ${ }^{4}$ These wrong solutions can probably not be prevented by reconfiguring the genetic algorithm. To find correct solutions in these problems, more information should be available. In many situations, more information is available about the sample than we included in the experiments described. For example, the composition of the carrier may be known. This kind of information must then be used to simplify the problem by limiting the number of parameters.

\section{CONCLUSIONS}

This paper presents a two-step fundamental parameter method for model-free analysis of thin layered materials by X-ray fluorescence spectrometry. The method is an improved version of the method described in ref 1 . A genetic algorithm is used to obtain the number of layers, for each layer the elements, and an estimate of the corresponding concentrations and layer thickness. The second step is a gradient technique to refine this estimate. Experiments are described on samples of various complexities. The experiments assume both an unknown number of layers and an unknown distribution of the elements present over the layers.

The circumscription of the method is the information present in the XRF intensities. It is shown that this information can be insufficient when the layers are too thin and/ or the sample is too 
complex. It is also shown, in the latter case that good results can be obtained when the angular dependence of X-ray fluorescence is measured. This is done by using two angles of detection. Our spectrometer is not equipped to measure at more than one angle. Therefore, the feasibility of the method for these types of samples is shown by means of simulated data. For relatively simple sample structures, measurements at one angle of detection yield sufficient information. Here, good results are obtained for both simulated and measured data.

\section{ACKNOWLEDGMENT}

We acknowledge the following contributions to the work presented: Philips Research for the financial support; Philip De Groot, Theo Reymers, Patrick Timmermans, and Alex Veldhuis, all (former) students at the K atholieke Universiteit Nijmegen, for their contributions to the development of the method presented; M irjan Bekkers, Philips Research Laboratories, Eindhoven, for fruitful discussions.

\section{SUPPORTING INFORMATION AVAILABLE}

Tables listing the measured/simulated intensities for all samples and the genetic algorithm configuration used in all experiments. This material is available free of charge via the Internet at http:/ / pubs.acs.org.

Received for review September 22, 1998. Accepted June 25, 1999.

AC981053Q 